\title{
A Taxonomy of Inference in Simulation Models
}

\author{
Thomas Brenner • Claudia Werker
}

Received: 26 July 2006 / Accepted: 12 July 2007 / Published online: 16 August 2007

(C) Springer Science+Business Media, LLC 2007

\begin{abstract}
Simulation models have become increasingly popular in economics in the last two decades, because they can deal with a wide range of research questions. The set-up and analysis of simulation models can range from very specific to very general and can be underpinned by different combinations of theoretical considerations and empirical data. We offer a taxonomy of existing simulation approaches and show how their results can be used to explain observed economic features, examine economic systems and predict future economic processes. Moreover, we offer a new type of method that helps to better exploit empirical findings in simulation models.
\end{abstract}

Keywords Methodology $\cdot$ Simulation models $\cdot$ Theory $\cdot$ Empirical data

JEL Classification B41 $\cdot$ B52 $\cdot$ C63

\section{Introduction}

In the last two decades simulation models have become increasingly popular in economics even though it is difficult to evaluate and compare their results. They have been used for various purposes by employing different modelling strategies and methods. This is, at least, partly caused by the fact that the simulation approach is compared to other methods very flexible. To give some examples, mathematical analysis is constrained to models that are treatable; econometrics is commonly used and restricted by the data that can be obtained for one kind of research unit; and experimental economics is limited by the types of situations that can be adequately reproduced in the lab. The simulation approach does not face such restrictions.

T. Brenner $(\bowtie) \cdot$ C. Werker

Max Planck Institute of Economics, Jena, Germany

e-mail: brenner@econ.mpg.de 
The flexibility of the simulation approach is both an advantage and disadvantage at the same time. The advantage is that simulation models can cover a much broader range of research questions compared with alternative methods. The disadvantage is that simulation models vary substantially_much more than models using alternative methods, which are usually based on core of assumptions agreed upon by the scientific community. These core assumptions are missing for simulation models. This does not only hinder their evaluation but also gets in the way of a fruitful discussion between scholars. Richiardi et al. (2006) correctly pointed out that there is a necessity to set up a standard for agent-based simulation models. They pointed at a range of potential pitfalls, such as the description of model structures or the interpretation of model behaviour. In our paper we do not advocate such a standard. We address the question what role should and can data play in simulation models. Moreover, for different kinds of simulation models, including agent-based models and microsimulations etc, we show how this issue affects the way the model is set-up, the runs are conducted and the results are interpreted.

To keep the advantage of flexibility of simulation models while at the same time deal with the disadvantage of ambiguity of simulation models we provide a taxonomy. This taxonomy is based on the use of empirical data, which can help to find the correct simulation model for a specific research question. First of all, we introduce three general principles of inference, i.e. induction, deduction and abduction (Sect. 2). Then, we develop a taxonomy of simulation models (Sect. 3). Based on that, we discuss the three scientific steps that are used in current simulation approaches and define a new type of simulation model, called abductive (Sect. 4). We conclude with a brief summary of our results (Sect. 5).

\section{Principles of Inference}

In preparation for the structured overview on simulation approaches some basic categories and principles of inference are described here. Simulation models roughly contain two components, i.e. implications and assumptions. Assumptions contain premises and definitions and set the boundaries for the model, e.g. for which kind of country it is set up or for which time period. Independent from the approach taken, simulations always provide the tool to derive implications from assumptions in an analytical and logical way. Running simulations means that we obtain knowledge about the implications of these assumptions, similar to the analysis of mathematical equations. Data can be used in both components of the model: In assumptions data helps build the model whereas in implications data is used to test the inferred implications. It is important to note that one cannot use the same data for assumptions and implications as this would lead to a self-evident model.

Three different principles of inference can be distinguished: deduction, induction and abduction. Deduction is often summarized as inferring "from general to particular" (cf. Lawson 1997, 24). Let us use, as an example throughout this paper, the impact of different patent laws on the development of certain industries in different countries. Deduction would mean that in this context we have or assume a theory about the development of industries, which depends on the national patent law, e.g. that stricter 
national patent laws protect innovators' property rights better and thereby gives more incentives to innovate. From this theory we could deduce that an industry in a country with lax patent laws innovates less compared to the same industry in a country with stricter patent laws. As one can readily see in this example, assumptions within the deduction already contain all information available. Generally spoken, deduction sustains information already contained in the assumptions but does not create a new one.

If $\mathrm{A}=\mathrm{B}$ and $\mathrm{B}=\mathrm{C}$,

(assumptions)

then $\mathrm{A}=\mathrm{C}$.

(implication)

When using deduction, assumptions contain all possible elements of the model, such as premises, definitions or causal relationship. Therefore, it is often claimed that models only using deduction are "true". However, this only holds in the sense that in these models implications are logically derived from the assumptions. It does not mean-as often implied - that the model correctly describes, explains and predicts economic processes. In this sense "true models" do not exist in economics, because there are virtually no assumptions that are unanimously accepted.

Induction is often summarized as inferring "from particular to general" (cf. Lawson 1997, 24). It starts from a description of some part of a population and then infers conclusions about the characteristics of the whole population. In our example this would mean that we observe the innovative output of a number of industries in a number of different countries with different patent laws. We would then inductively infer general mechanisms, relationships and rules by examining the common characteristics of all observations and could come to the conclusion that industries in countries with stricter patent laws have a larger innovative output. As the inductive principle runs "from particular to general" it is often seen as creating information-however doubtful. The inference in induction states something that is not contained in the original observations. If the inference arguments are strong, it is probable that the claims made about the conclusions hold. Inductive inference is based on data. However, even if the number of observations in the data set is large, it is in principle impossible to have all observations available, not in the least because future events cannot be observed. This means that the implications derived from data are uncertain. In the future, the same will happen only with an unknown probability. This probability is impossible to gain, because future observations, by definition, cannot be made now.

Abduction-sometimes also called retroduction-categorises "particular events into general patterns" (Lawson 1997, 24). In our example abduction means that we argue that industries vary in their development and are therefore affected by patent laws in different ways. Abduction means that we start by collecting detailed information about the development of various industries in different countries facing different patent laws. Based on this, we categorise the different developments and identify the underlying driving forces. This also enables us to describe, explain and predict developments of industries in other countries with respect to their patent laws. It is important to note that abduction requires data based on substantial and detailed observations. Only then is it possible to find meaningful and sensible underlying mechanisms to infer from the assumptions to the implications. So, for example, if we observe that a number of low-tech industries develop in different countries independent of actual 
patent laws, we might conclude that all low-tech industries are not influenced by any change in the patent laws. Obviously, this conclusion has been drawn too quickly. Abduction requires much more detailed information to infer implications that are likely to hold when confronted with reality. In our example one would wish to know much more about the mechanisms behind the industrial development and the differences between national patent laws. It would especially be important to know what makes an industry's development independent from patent laws. We could for instance choose to define categories of industries that show similar developments within one category and different developments between categories, e.g. the famous Pavitt taxonomy (Pavitt 1984). By going back and forth between theorizing and empirical testing we might come to the conclusion that science-based industries profit more from stricter patent laws than scale intensive industries. This would allow transferring the experience to other industries. As more relevant data details are known they can be precisely categorised into a general pattern.

Abduction enables us to identify underlying structural elements that explain observations, and to develop a theory of the part of the world we are investigating. This takes us a substantial step further than pure deduction or induction, because abduction helps us to connect theory and data in a creative way. By using the principle of abduction we are able to create new information. According to Peirce (1867/1965, 5, 145f):

“(Induction) never can originate any idea whatever. No more can deduction. All the ideas of science come to it by the way of abduction. Abduction consists in studying the facts and devising a theory to explain them. Its only justification is that if we are ever to understand things at all, it must be in this way."

If one looks at abduction this way it becomes obvious that abduction is a crucial step in theory formation and validation in general (Thagard 1988) because it helps to find the best possible explanation for processes in the real world.

\section{A Taxonomy of Simulation Models}

\subsection{Principles of Classification}

Simulation models differ considerably in the way their assumptions are set up. We offer a taxonomy of simulation approaches with two axes that represent two crucial features of simulation models. First of all, simulation models can range from very specific to very general. Second, different combinations of theoretical considerations and empirical data can underpin the assumptions used. With respect to the degree of generalization of the simulation model we distinguish between 'general' and 'specific' analyses. In specific analyses only one specification of the simulation model is extensively simulated and the results analysed. This implies that the modeller implicitly assumes the possibility to design the model and fix the parameters adequately on the basis of theoretical considerations or empirical knowledge about the underlying mechanisms of the simulated dynamics. Only few approaches are that extreme. Often certain parameters are varied to conduct counter-factual analyses or to examine the robustness of the results. Sometimes different model specifications are examined in 


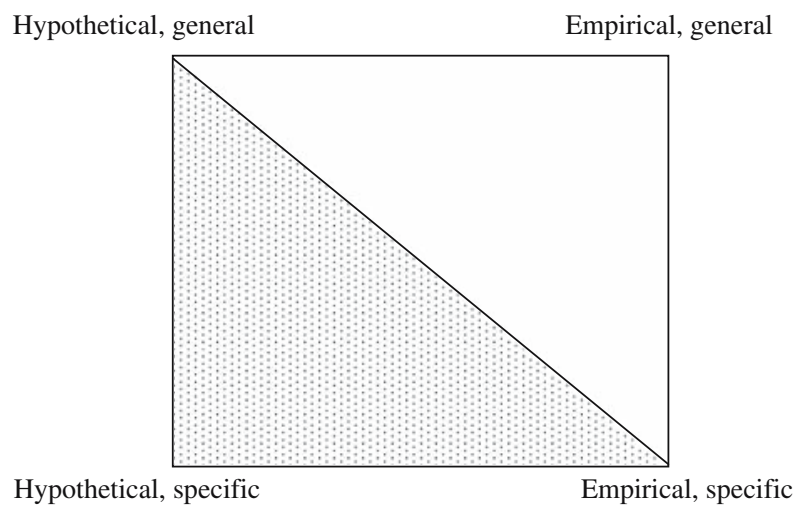

Fig. 1 Classification dimensions of simulation models

order to compare them. The other extreme would be to analyse an infinite number of different model specifications. This means that the parameters of the model remain unspecified and that the simulation is run for all different combinations of parameter values. An even more extreme approach would be to also include in the analysis all different kinds of model set ups that are possible according to some considerations. Such an approach would be called 'general' here. Of course, a completely general approach is not feasible because it would require an infinite number of simulation runs. Nevertheless, there are simulation approaches in which a large number of different model specifications are examined. Such an approach is used, for example, in Bayesian simulations, where inferring knowledge about the parameters from empirical data about the system's dynamics is the main goal. The specificity-generality dimension is used as one axis (see Fig. 1).

The second dimension of Fig. 1 concerns the use of theoretical considerations and empirical data. At one extreme end are simulation models that are set up without any use of empirical data, based on theoretical considerations or ad-hoc assumptions. At the other end of the spectrum all model specifications, meaning all parameter values and the model's set-up, are based on empirical data. We call the former a 'hypothetical' model and the latter an 'empirical' model. Again, the two extremes do not emerge in reality. While it is in principle possible to carry out a purely 'hypothetical' approach, usually all models are based on at least some empirical knowledge-in the form of general experiences, stylised facts, or common empirical knowledge in the discipline. A model based on every empirical detail is not possible because the collection of empirical data already includes theoretical considerations and so does the combination of empirical data in order to set up assumptions for a model. We use the hypothetical-empirical distinction as the second axis of Fig. 1. It denotes the amount of empirical data used to specify the simulation model.

When setting up assumptions for simulation models the ultimate aim is to learn something about the underlying dynamics that drive the real world. In this context, simplification is a major tool, because modellers are usually not interested in all features and dynamics of economic processes but only in those relevant for the research question(s) under investigation. This affects the way assumptions are set up. For the 
first dimension of the classification of simulation models in Fig. 1, one can offer a general rule: The more general a model is, the more it is able to cover a larger variety of economic processes. This implies that a more general approach, covering more different model specifications, is more likely to adequately represent a specific dynamic in the real world with a particular specification. With respect to the use of empirical data, one can provide the following general rule: The more complex and complicated the economic processes captured by the model are, the more empirical data needs to be used to adequately restrict the model parameters.

Models above the diagonal (from hypothetical, general to empirical, specific) are not possible because this would imply completely fixing parameters according to empirical data and, nevertheless, examining all different parameter and model settings. This is a contradiction because examining different settings means that they are not fixed. Hence, a simultaneously completely empirical and completely general approach does not exist. Only approaches below the diagonal are feasible.

\subsection{Discussion and Classification of Simulation Models}

In the following, we will classify existing simulation models and define a new category, which we call the abductive simulation model. Simulation approaches vary in many ways, e.g. regarding how they embed the simulation in the overall analysis, regarding how many different simulation models are analysed, regarding how assumptions are obtained and how the implications are interpreted. We will classify the simulation models according to the hypothetical-empirical and general-specific dimensions summarized in Fig. 1 (see Sect. 3.1) and according to way they use and combine different inference principles (see Sect. 2).

The first type of approach is taken by most scholars using the simulation method and is called this the conventional simulation approach. In conventional simulation approaches specific models are analysed and data is used in the form of stylised facts on the resulting level to check the implications. The assumptions in the simulation models are based on theoretical considerations, which result from axioms, ad-hoc modelling or stylised facts. Stylised facts are used in accordance with Kaldor's original idea (Kaldor 1968, 177f). They comprise empirical statements about a wide application area. They mostly rely on common sense and the impression of the scholar using them. The problem with stylised facts is that they "fall from heaven" and often remain unmotivated (for a detailed critique see Schwerin 2001, 92-98). As it is usually unclear how stylised facts are derived it is not possible to tell whether or not they comprise only the structural elements of economic processes or whether they partly mirror noise in the form of chance elements. Nevertheless, the use of stylised facts enables modellers who want to concentrate on specific theoretical considerations to integrate some empirical reality into their analysis. At the time they are set up conventional models are located in Fig. 1 rather in the lower left edge (see Fig. 2). The major principle of inference used in this kind of model is deduction, because the focus lies on theoretical considerations and the analysis of their implications. This deduction is conducted through simulations. Usually, there is also some induction involved because computational economists often check their models in the light of common, (usually empirical) knowledge about 


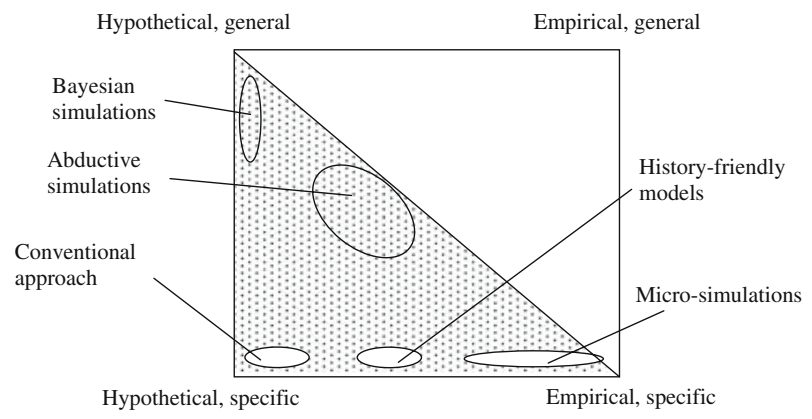

Fig. 2 Classification of five different types of simulation approaches

features of reality that correspond to the simulation results. If fundamental differences are detected, the simulation model is revised to eliminate these differences. The idea is to find a simulation model that shows a behaviour that corresponds to the expectations based on common knowledge. This inductive step is, however, usually not presented in the publication. Only the final simulation model is presented and analysed.

Examples of simulation models, which use stylised facts in their modelling, are Harrison (2004), Fagiolo and Dosi (2003), Werker (2003), Winter et al. (2003) and Windrum/Birchenhall (1998). Harrison (2004) presents a simulation model that can reproduce stylized facts in the form of four different kinds of organizational evolution and firm growth. Fagiolo and Dosi (2003) present an endogenous growth model, which is able to reproduce stylized facts in the form of empirically plausible GDP time-series. Windrum and Birchenhall (1998), Winter et al. (2003) as well as Werker (2003) present models, which are able to account for the stylized facts in the evolution of industry life cycles. Harrison (2004), Fagiolo and Dosi (2003), Werker (2003) and Windrum and Birchenhall (1998) model random effects and perform a sensitivity analysis by running the same specifications at least 100 times. They calculate some statistics and give qualitative impressions of these results. Winter et al. (2003) concentrate more on the analytical results of their model and limit themselves to only a few calculations to test for the sensitivity of their results.

The second type of approach is the history-friendly model, i.e. specific models, which use case study data. They rely on detailed empirical knowledge about real historical processes and try to find a model that leads to processes with the same characteristics. These models focus mainly on induction, because the case study is the core of the analysis. The case study is used in two ways. On the one hand, it provides some knowledge about the underlying processes that are modeled in the simulation. On the other hand, it provides information about the realistic dynamics of the simulation. Hence, the model uses quite some empirical data. Once a simulation model is found that describes the historical case adequately, simulations are run in order to study various aspects of interest. This is a deductive endeavor, for which a specific setting is chosen as the basic model. The approach is therefore located in the lower middle of Fig. 1.

A problem of this approach lies with the concentration on one historical example. Although usually most of the complicated and complex processes involved are 
depicted, scholars using history-friendly models have difficulties in identifying the underlying general mechanisms, which could be found back in similar historical circumstances. To some extent, carrying out sensitivity analyses of the results can solve this problem, because this gives an indication of how stable the results are and their dependence on random effects. Generally, history-friendly models share the advantage as well as the disadvantage with case studies: they give us deep inside knowledge about real economic processes but lack the possibility to generalize their results in a convincing way.

Recent examples of history-friendly models are Eliasson et al. (2004), Kim and Lee (2003), Malerba and Orsenigo (2002), Eliasson and Taymaz (2000) and Malerba et al. (1999). Eliasson et al. (2004) show, with an empirically calibrated micro-macro model for Sweden, how the new economy affects productivity and growth processes. Kim and Lee (2003) construct a history-friendly model for the DRAM industry. Malerba and Orsenigo (2002) and Malerba et al. (1999) concentrate on analyzing a history-friendly model of the computer-industry. Only Eliasson and Taymaz (2000) examine the sensitivity of their results. All other authors run a number of counterfactual histories in order to account for the sensitivity of their results. Thereby, they produce data that can be statistically analyzed. Consequently, these scholars can use more sophisticated methods and can look into the statistical properties of their models in a systematic way. This gives some insights into how counterfactual histories could have occurred and on how much the results depend on random effects.

The third type of approach is micro-simulations, using specific models, which are based on comprehensive empirical data. In these models the dynamics of the real system is thoroughly examined. Statistical methods are applied to detect the crucial dependencies between variables and the trends in the dynamics of these variables. Two requirements have to be satisfied when carrying out micro-simulations. First, the dynamics that are studied have to be so simple to be sufficiently analysed. Second, adequate empirical data has to be available to examine the underlying mechanisms. Given that these requirements are satisfied, the obtained simulation model can be used to describe, explain or predict economic processes. The findings lead to a simulation model that describes the previously observed dynamics. The crucial theoretical assumption is that, in the future, the same mechanisms and dynamics take place as in the past. The problem is that this is not necessarily the case. In Fig. 2 this approach is located in the lower right edge because a highly specialised model is developed using a large amount of empirical data. Micro-simulations use induction extensively to set up the simulation model, while subsequently deduction is used to make predictions and counter-factual analysis with the help of simulations.

Micro-simulation approaches can be frequently found in the literature (surveys and general discussions can be found, e.g., in Merz 1991, O'Donoghue 2001, and Creedy and Duncan 2002). They typically either aim at investigating the effect of certain policies or at predicting future developments. For example, Atkinson et al. (2002) study the impact of a European Minimum Pension. To this end, they use a simulation model that is based on detailed data about household income in the five largest EU countries and simulate the impact of a European Minimum Pension on these incomes. Based on similar data for the UK and a similar simulation approach, O'Donoghue and Sutherland (1999) study the impact of alternative family tax treatments on the tax paid 
by these families and work incentives. The different approaches of various European countries are compared on the basis of the situation in the UK.

The fourth kind of approach is the Bayesian simulation approach, using general models, which incorporate empirical data by systematically comparing the simulation results to larger sets of empirical observations. This approach starts from the assumption that little is known about the models' parameters and often also about the relationships between the variables of the model. The large set of empirical observations, which is typically used, enables the modeller to thoroughly test the different specifications of the simulation model. In Bayesian simulations usually a lot of empirical data is available for the phenomenon but little for the processes that cause this phenomenon. Therefore, a very general simulation model is developed that includes all plausible processes that could cause the phenomenon, so that the initial simulation model is located in the upper left edge in Fig. 2. The intention of Bayesian simulations is to inductively obtain an adequate model specification. The model specification obtained might then be used for further analysis in a deductive way, but this is not the major part of this approach.

Bayesian inference has become increasingly widespread in econometrics in recent years (for an introduction to Bayesian inference see, e.g., Citro and Hanushek (1991) and the original work by Zellner (1971)). Examples for Bayesian simulations are, Kaufmann (2000), Tsionas (2000), Jacobson and Karlsson (2004) and Jochmann and Leon-Gonzalez (2004). Tsionas (2000) uses stock market data to check different models about the stochastic movements of prices. He simulates a number of existing models with different parameters. Because of the stochastic dynamics of the model it is run numerous times for each specification and the frequency of correspondence with empirical observations is counted. According to Bayesian inference, this offers some knowledge about the likelihood that each of the parameter specifications and each model are correct. This allows for judging the adequateness of various existing models. A similar approach is taken by Kaufmann (2000). Bayesian simulations, however, can also be used to make predictions about future developments (see, e.g., Jacobson and Karlsson, 2004 and Jochmann and Leon-Gonzalez 2004). Jacobson and Karlsson (2004) use the Bayesian approach to evaluate the relevance of certain potential indicators in forecasting inflation in Sweden. This resulting knowledge can then be used to make predictions. Jochmann and Leon-Gonzalez (2004) used the Bayesian principle to estimate the parameters and features of a model which predicts the demand for health care by classifying the population into classes according to their health status.

The fifth kind of approach is the abductive simulation model, using simulations in which empirical data is used as much as possible to specify the model, while keeping the model as general as necessary. In this kind of model, one major aim is to develop a model that adequately represents the economic processes, which are supposed to be analyzed by the model. Abductive simulation models start, similar to Bayesian simulations, with the set up of a very general model that should represent all mechanisms and processes that might play a role for the considered research question. In contrast to Bayesian simulations, empirical knowledge about the modeled mechanisms and processes is then immediately used to reduce the generality of the model. However, it is crucial in this approach that the generality is reduced only as far as empirical data allows. For example, if empirical evidence is available that shows that 
certain parameters fall into certain ranges, these ranges are used to restrict the model parameters. As a consequence, the simulations are started with a model based on some empirical knowledge but are still quite general and therefore located in the middle of Fig. 2. Because of the model's generality, many different specifications have to be run, as done in the Bayesian approach. Furthermore, empirical data about the real characteristics and dynamics of the modeled system is also used, as in Bayesian simulations. However, it is not used to find the best specification of the model, but only to eliminate those specifications that are unable to produce the real characteristics and dynamics of the system. This means that empirical data about the system's behavior is used to further reduce the model's generality. Nevertheless, the model is usually not completely specified. It is argued that a set of model specifications remains that are all potential candidates for adequately representing reality.

The obtained model specifications of abductive simulation models are finally used to answer the research question, which might be about predictions, relationships, counter-factual developments, or causal mechanisms. Only answers that are obtained for all studied model specification, which have to be picked randomly from the set of remaining model specifications, are considered to be reliable. This approach does not only combine induction and deduction when setting-up the model and when carrying out the runs. It takes an additional abductive step by using empirical data about specific systems to reduce the generality of the model and by employing the resulting set of model specifications to generate knowledge about the kind of systems that are studied. It is important to note that in the sense of Thagard (1988) many scholars design simulation models in an abductive way, because they try to infer the best possible model from theoretical considerations and/or empirical data. In this context Richiardi et al. (2006) correctly point at the fact that the logic behind agent-based simulation models is abductive. However, in contrast to the here suggested abductive simulation models these models do not provide a transparent discussion of the abductive step. In particular, they do not explicitly use empirical data for both designing assumptions and testing implications of the model to make this abductive step visible.

Initial elements of this approach were first used in Brenner (2004, Ch. 4) in order to develop knowledge on the emergence of local clusters. Various economic processes involved in the emergence of local clusters were modeled by defining ranges of values for all parameters on the basis of empirical data. The characteristics of local cluster formation were studied for many different parameter settings. Thus, the first step proposed for abductive simulation models to use as much empirical data as possible when setting-up the assumptions were taken. However, the subsequent abductive step was not included. This step was done to some extent in the simulation approach formalized and applied for the synthetic dye industry by Brenner and Murmann (2003), where the empirical data available for the industry's development from 1856 to 1913 were used. Again ranges for all parameter values have been defined and simulation runs were conducted accordingly. With their model Brenner and Murmann (2003) analyzed the market shares of the firms in the various countries, namely France, Germany, Switzerland, UK, and USA, in 1913. The real values of these market shares were confronted with the results for the different parameter settings that had been run. All parameter settings that produced the observed market shares were used to study the characteristics of the economic system and conduct counter-factual analysis. Thus, 
Table 1 Inferring results in simulation models

\begin{tabular}{|c|c|c|c|c|}
\hline \multirow[t]{2}{*}{ Approach } & \multirow{2}{*}{$\begin{array}{l}\text { Building the } \\
\text { model }\end{array}$} & \multirow{2}{*}{$\begin{array}{l}\text { Conducting the } \\
\text { runs }\end{array}$} & \multicolumn{2}{|c|}{ Analysing the results } \\
\hline & & & Usually done & Possible \\
\hline Conventional & $\begin{array}{l}\text { Rather } \\
\text { hypothetical, } \\
\text { specific }\end{array}$ & $\begin{array}{l}\text { Usually one } \\
\text { specification run }\end{array}$ & $\begin{array}{l}\text { Characteristics } \\
\text { study }\end{array}$ & Comparison \\
\hline Microsimulations & Empirical, specific & $\begin{array}{l}\text { One specification } \\
\text { run }\end{array}$ & $\begin{array}{l}\text { Prediction, } \\
\text { characteristics } \\
\text { study, some } \\
\text { comparison }\end{array}$ & - \\
\hline Bayesian & $\begin{array}{l}\text { Hypothetical, } \\
\text { general }\end{array}$ & $\begin{array}{l}\text { Many } \\
\text { specifications run } \\
\text { (sometimes } \\
\text { Monte-Carlo) }\end{array}$ & Comparison & $\begin{array}{l}\text { Characteristics } \\
\text { study, Prediction }\end{array}$ \\
\hline History-friendly & $\begin{array}{l}\text { Rather empirical, } \\
\text { specific }\end{array}$ & $\begin{array}{l}\text { One specification } \\
\text { run, with } \\
\text { sensitivity } \\
\text { analysis }\end{array}$ & $\begin{array}{l}\text { Comparison, } \\
\text { characteristics } \\
\text { study }\end{array}$ & - \\
\hline Abductive & $\begin{array}{l}\text { As empirical as } \\
\text { possible, as } \\
\text { general as } \\
\text { necessary }\end{array}$ & $\begin{array}{l}\text { Many } \\
\text { specification runs } \\
\text { (Monte-Carlo) }\end{array}$ & $\begin{array}{l}\text { Comparison, } \\
\text { characteristics } \\
\text { study, } \\
\text { categorisation }\end{array}$ & Prediction \\
\hline
\end{tabular}

here the abductive step was taken and knowledge about the underlying economic processes driving market shares were derived. In particular, Brenner and Murmann (2003) found that the development of the dye industry depended severely on two factors, i.e. the university system's responsiveness and the availability of chemists.

\section{Inferring Results in Simulation Models}

Most simulation approaches can be separated into three steps, which are usually conducted consecutively: first, computational economists build the simulation model by defining the assumptions (Sect. 4.1 and Table 1, second column). Second, they conduct the simulation runs after having determined the parameter values (Sect. 4.2 and Table 1, third column). Finally, they analyse the simulation results in order to describe, explain, understand, and/or predict economic processes (Sect. 4.3 and Table 1, last two columns).

\subsection{Set up of Simulation Model}

The simulation method might be used in modelling in various ways. To make the following discussion more comprehensive, we call all restrictions, definitions and settings on the level of the simulation model 'assumptions' - also if they result from an empirical estimation or the fitting of the simulation model to data about the dynamics 
of the real system. Similarly, we call features and dynamics that result from running the simulation 'implications'. This is done to make clear that two levels are involved in a simulation approach: the level on which the model is built, i.e. the assumptions, and the level on which the results of simulations are analysed, i.e. the implications.

In the first step of a simulation model the assumptions are determined and the model is set up. The assumptions stem either from ad-hoc judgements, theoretical considerations or empirical data. Ad-hoc judgements are difficult to evaluate. Unanimously agreed assumptions stemming from theoretical considerations are rare. That is why we argue for the use of as much empirical data as possible. This can consist of all kinds of data from panel data to case studies (cf. Moss and Edmonds 2005). This would lead to simulation models that are not only more widely accepted in the scientific community, but also to models that would more reliably describe, explain and predict economic processes.

Empirical data can be available in the two components of a simulation model, i.e. the assumptions that are used for building the simulation model and the implications that are studied as the outcome of the simulation runs. The same data set, of course, can not be used for both components. However, empirical data about the assumptions of the simulation model and other empirical data about the implications of the simulation model can be used in one approach to make the model representing economic processes more adequate. In the following, we discuss how empirical data can be used to build the model. In Sect. 4.3 we discuss how empirical data is used within the model's implications.

The use of empirical data from different levels is in line with Critical Realism, which we suggest as an appropriate methodological basis for simulation models. Protagonists of this school of thought recognise that the world is structured into different layers (Downward et al. 2002). They aim at describing and explaining empirical facts in terms of their underlying structures categorizing them into theoretical findings and deriving causal explanations from the analysis. Critical Realists basically approach empirical data the way scholars carrying out case studies do and therefore face the same kinds of problems: Data collected and analysed lack the potential to generalize results. To overcome this problem we have to compare larger sets of cases in a systematic way and to identify what they have in common independent of their specific historical circumstances. In a first attempt to do so Brown et al. (2002) suggested combining Critical Realism with "systematic abstraction" as a means to achieve a historical level of generality and to identify the inner connection of social phenomena. However, they do not provide a guideline how to put their suggestion into practice. Because of their flexibility, simulation models are the perfect tool to put their suggestion to use. In Sect. 4.3, we will show how the use of empirical data in the so-called abductive simulation model helps to categorize empirical findings into classes of models.

Empirical data is often either not available or difficult to obtain. Thus, to develop a completely empirically based model, as used or intended in micro-simulations, is not feasible in, at least, most cases. As a consequence, we have to answer the question of how to deal with insufficient empirical data. On top of ad-hoc judgements or theoretical considerations there is an alternative option, which is usually neglected in the literature, i.e. the simulation model can be kept as general as necessary. This procedure is in line with the inference concept of abduction, because for all aspects in which we 
can not or do not want to use empirical data to specify the simulation model, we keep the model general. In practical terms this means that we include all logically possible values for those parameters for which we cannot fix the value or restrict the range of values. The same holds for the set up of the model. If empirical data does not allow us to choose one of two alternative model settings, we examine both of them. Of course, some simplifications are necessary, because otherwise we will never obtain a treatable model. However, these simplifications should only affect features of the model that can be reasonably assumed to be less important for the topic of the study. In case of doubt we argue in favour of keeping the simulation model general.

\subsection{Conduction of Simulation Runs}

The second step is the conduction of the simulations. Once the assumptions are defined and the simulation model is built, simulations can be run. Differences between approaches in this step stem from technical differences as well as from the way simulation results are analysed. Discussing the technical differences stemming from using various computer languages and/or simulation platforms would go way beyond the scope of this paper. In the following, we concentrate on differences caused by various ways of analysing the results.

Simulation results are analysed in various ways so that simulation runs are conducted differently. An analysis of the robustness of the results with respect to some parameters requires that the simulation has to be run for various values of these parameters. For more general models simulations have to be conducted for all possible values of these parameters. Usually some parameter ranges can be defined (a comprehensive discussion can be found in Werker and Brenner 2004). Nevertheless, this usually implies an infinite number of possible values for parameters (if for logical reason we do not deal with full numbers), so that not all possible values can be simulated. A Monte-Carlo approach is used in such a case, meaning that repeatedly parameter values are randomly picked from their range and simulations are run for these values. The more simulations are run, the more likely an adequate parameter set is used, in at least one simulation run.

There is a trade-off between the empirical calibration and generality of the model. Let us consider a situation in which a number of parameters are crucial for the behaviour of a simulation model and their values determine whether we obtain reliable answers for our research question. In a completely general approach all these parameters remain unrestricted and a Monte-Carlo approach is taken. We can then be quite sure that, at least, one simulation run is conducted with an adequate parameter set if we run many different parameter sets. We might also restrict the parameters to certain ranges by using empirical data or logical arguments. We argue that an ad-hoc fixing of parameters should be avoided. Furthermore, only uncontroversial theoretical arguments should be used. Even if empirical data is used, a fitting of parameters to one value is rarely feasible, because the parameters are usually statistically estimated. Exeptions are, for example initial values, such as the number of chemists in 1856 in the simulation of the synthetic dye industry by Brenner and Murmann (2003), that can be taken from historical records. For all other parameters a range of values has to be 
considered and whilst interpreting the result it has to be kept in mind that only one value in this range represents an adequate choice.

The collection of empirical data for the set up of a simulation model is usually very cumbersome. The use of empirical data can be substituted by keeping the model more general. However, this has two impacts. First, more simulations have to be run because there are more possible parameter sets. This increases the necessary computer time. Second, only one of these many simulation runs might be adequate for answering the research question, and we do not know which simulation run has this characteristic. As a consequence, only those results are reliable that result from all simulation runs for all possible parameter sets. We obtain, in general, fewer reliable results than with a more specific simulation model.

\subsection{Use of Simulation Results}

In the third step the simulation results are used to answer the research question. The analyses that are conducted depend on the research question addressed and the type of approach employed. We distinguish between three kinds of research questions in this context: examining characteristics of economic processes modeled including counterfactual analyses, predicting economic processes, or comparing simulation results with empirical data. In line with these different research aims, we distinguish three typical ways of inferring scientific insights from the results of simulation models, which use a combination of induction and deduction. In addition we include a fourth one that explicitly uses abduction as inference principle by categorizing simulation model specifications into sets of models.

\subsubsection{Characteristics Study}

In many simulation approaches the simulation results are used to study the characteristics of the system that has been modelled. The aim is to understand the relationship between variables and/or parameters or to conduct counter-factual analyses. This implies that the simulation results are treated similar to empirical data. Sometimes the resulting dynamics or characteristics are simply described. Sometimes they are analysed with the help of statistical tools. This allows for the examination of the (causal) relationships between parameters, initial conditions, or specifications of the simulation model and the dynamics or outcomes of the simulation runs. The aim is to obtain detailed knowledge about the system described by the simulation model. Whether valid knowledge is obtained depends crucially on the adequateness of the simulation model.

Such analyses are frequently conducted in the conventional and the history-friendly approach. Although the micro-simulation approach is mainly used to make predictions, some counter-factual analyses are also conducted. In the abductive simulation model, the examination of both, parameter relationships and the systems characteristics is also part of the main task. However, before this task can be tackled a comparison must be conducted. We discuss this two-step procedure for using the simulation results below. 


\subsubsection{Prediction}

Predicting future developments on the basis of simulation runs employs deduction. Naturally, its quality depends crucially on the quality of the simulation model. In the literature predictions are mainly carried out by a micro-simulations approach, because they are built on the basis of a comprehensive data base. Hence, the predictions gained can be assumed to be quite accurate. However, other approaches that use a lot of empirical data to test their implications, such as the Bayesian and the abductive simulation models, would provide predictions of the same quality.

\subsubsection{Comparison between Simulation Results and Empirical Data}

It is also quite common to compare the simulation results with empirical data about the system's dynamics and characteristics. The aim is to identify the underlying causal relationships to explain known phenomena. Potential explanations are formulated as assumptions in the set up of the simulation model and are tested by comparing their implications (simulation results) with empirical data. The approaches that use such comparisons vary substantially. One way is to simply use stylised facts to check whether the simulation results are plausible. This is done in many conventional approaches and in history-friendly models. Alternatively, Bayesian simulation models statistically compare the results of various simulation runs with empirical data. The aim is to identify those model settings for which the simulation results are in line with the empirical data. Other approaches that aim to predict future events, such as microsimulations, sometimes compare simulation results with empirical data to check the adequateness of their simulation model.

\subsubsection{Categorisation into Systems of Models}

In addition to the usual steps described above, we add a potential fourth step of categorising the simulation model specifications into sets of models. This fourth step is based on the abductive simulation approach. In this approach the available empirical data on the assumption level is used to specify the simulation model as much as possible. Then, with the help of a Monte-Carlo approach and a comparison of simulation results with empirical data on the resulting (implication) level, the model is further specified. Thus, the resulting model can be used for the different analyses discussed above. Alternatively, if empirical data is available to test the results for different real systems, these real systems can be grouped in the different categories. As the analysis can be carried out for each category of systems, we obtain different specifications of the simulation models that refer to different groups of real systems. The characteristics of the different specifications can be analysed and compared. Eventually, the resulting model specifications categorize the real system thereby giving us more detailed insights into the functioning of the economic processes modeled (see Werker and Brenner 2004 for a description of this procedure). In this way, we obtain classes of systems and knowledge about their characteristics and dynamics. Here, we truly employ abduction and we believe that this is very helpful for understanding economic systems. Obviously, simulations are very adequate tools to support this logical process. 


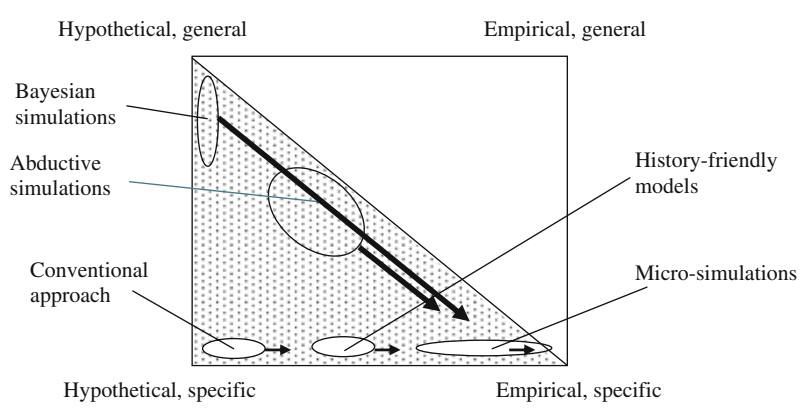

Fig. 3 Classification of simulation models after empirically checking implications

Testing the simulation results with empirical data, independent of how this is done, increases the amount of empirical data used for the specification of the simulation model. Therefore, every testing of a model's implications moves the model more to the right side of Fig. 3. In the Bayesian and the abductive approach empirical data on the implications of the simulation model are extensively used to specify the model further. In the other approaches only some comparison is sometimes conducted and the results are mainly used to test the model and revise it in case of negative results.

\section{Conclusions}

The taxonomy we provide in this paper sheds light on the role that empirical data play in simulation models. We distinguish existing simulation approaches according to the extent to which they use empirical data and according to the model specifications analyzed. Moreover, we provide an explicit overview of the steps that are and can be conducted in a simulation approach. This enabled us to stress the advantages and disadvantages of various simulation approaches in the light of the different ways in which the steps are taken.

We suggest a new type of model that we call abductive simulation model because abductive inference is not only at the core of these models but also made visible in the presentation of the model. This is notwithstanding the fact that many models implicitly use abduction as inference principle. Abductive simulation models include as much data as possible when setting up the assumptions and when testing the implications of the model. Assumptions are either specified by using empirical data or by including all logically possible model specifications. One needs to be aware of the trade-off between using empirical data and analyzing a lot of model specifications. Assume that we investigate the same research question once including comparably much and then including comparably little empirical data into our simulation model. For the model with more data we can estimate the parameters of the model and define quite small ranges for them. It is very likely that the adequate values of the parameters, i.e. the values that best represent the real world captured by this model, are within these small ranges. If we want a similar likelihood for including the adequate values for the model with less data we need to keep the ranges of the parameters much bigger. This, 
however, implies that many runs must be conducted to investigate the implications for the whole parameter ranges.

Generally, there are two main conclusions that we draw for all kinds of simulation models. First, we argue that empirical data should be used more often where it is available, because this leads to much better founded simulation models and increases the soundness of the results. Second, simulation models seem to be the perfect tool to transfer (empirical) knowledge from the level of assumptions to the level of implications and vice versa. This means that in a simulation approach one can go forth and back between the assumptions and implications in the simulation model and identify the characteristics and dynamics of the economic processes modeled. Empirical data used for setting up assumptions and for testing implications improve the comprehension of the economic processes modeled. Even a categorization of systems on the basis of abduction is possible when using abductive simulation models.

We argue that simulation approaches offer scientific potentials that are not fully exploited yet when using abduction. In particular, abductive simulation models have the potential to be widely accepted in the scientific community, because they would better describe, explain and predict economic processes.

\section{References}

Atkinson, T., Bourguignon, F., O’Donoghue, C., Sutherland, H., \& Utili, F. (2002). Microsimulation of social policy in the European Union: Case study of a European minimum pension. Economica, 69, 229-243.

Brenner, T. (2004). Localised industrial clusters: Existence, emergence and evolution. London: Routledge.

Brenner, T., \& Murmann, J. P. (2003). The use of simulations in developing robust knowledge about causal processes: Methodological considerations and an application to industrial evolution. Max-Planck-Institute of Economics, Jena, Papers on Economics \& Evolution, mimeo: \#0303.

Brown, A., Slater, G., \& Spencer, D. A. (2002). Driven to abstraction? Critical realism and the search for the 'inner connection' of social phenomena. Cambridge Journal of Economics, 26, 773-788.

Citro, C. F., \& Hanushek, E. A. (Eds.). (1991). Improving information for social policy decisions: The uses of microsimulation modeling I, review and recommendations. Washington DC: National Academy Press.

Creedy, J., \& Duncan, A. (2002). Behavioural microsimulation with labour supply responses. Journal of Economic Surveys, 16(1), 1-39.

Downward, P. J., Finch, H., \& Ramsay, J. (2002). Critical realism, empirical methods and inference: A critical discussion. Cambridge Journal of Economics, 26, 481-500.

Eliasson, G. D., Johansson, D., \& Taymaz, E. (2004). Simulating the new economy. Structural Change and Economic Dynamics, 15(3), 289-314.

Eliasson, G. D., \& Taymaz, E. (2000). Institutions, entrepreneurship, economic Flexibility and growthexperiments on an evolutionary micro-to-marco model. In U. Cantner, H. Hanusch \& S. Klepper (Eds.), Economic evolution, learning, and complexity (pp. 265-286). Heidelberg: Springer-Verlag.

Fagiolo, G., \& Dosi, G. (2003). Exploitation, exploration and innovation in a model endogenous growth with locally interacting agents. Structural Change and Economic Dynamics, 14, 237-273.

Harrison, J. R. (2004). Models of growth in organizational ecology: A simulation assessment. Industrial and Corporate Change, 13(1), 243-261.

Jacobson, T., \& Karlsson, S. (2004). Finding good predictors for inflation: A Bayesian model averaging approach. Journal of Forcasting, 23, 479-496.

Jochmann, M., \& Leon-Gonzalez, R. (2004). Estimating the demand for health care with panel data: a semiparametric Bayesian approach. Health Economics, 13, 1003-1014.

Kaldor, N. (1968). Capital accumulation and economic growth. In F. A. Lutz \& D. C. Hague, (Eds.), The theory of capital. Proceedings of a Conference held by the International Economic Association (1958) (pp. 177-222). London: Macmillan Press. 
Kaufmann, S. (2000). Measuring business cycles with a dynamic Markov switching factor model: An assessment using Bayesian simulation methods. Econometrics Journal, 3, 39-65.

Kim, C. W., \& Lee, K. (2003). Innovation, technological regimes and organizational selection in industry evolution: A 'History Friendly Model' of the DRAM industry. Industrial and Corporate Change, 12(6), 1195-1221.

Lawson, T. (1997). Economics and reality. London: Routledge.

Malerba, F., Nelson, R., Orsenigo, L., \& Winter, S. (1999). 'History-friendly' models of industry evolution: The Computer Industry. Industrial and Corporate Change, 8, 3-40.

Malerba, F., \& Orsenigo, L. (2002). Innovation and market structure in the dynamics of the pharmaceutical industry and biotechnology: Toward a History-Friendly Model. Industrial and Corporate Change, 11, $667-703$.

Merz, J. (1991). Microsimulation-a survey of principles, developments and applications. International Journal of Forecasting, 7, 77-104.

Moss, S., \& Edmonds, B. (2005). Towards good social science. Journal of Artificial Societies and Social Simulation, 8(4).

O’Donoghue, C. (2001). Dynamic microsimulation: A methodological survey. Brazilian Electronic Journal of Economics, 4(2).

O'Donoghue, C., \& Sutherland, H. (1999). Accounting for the family in European income tax systems. Cambridge Journal of Economics, 23, 565-598.

Pavitt, K. (1984). Sectoral patterns of technical change: Towards a taxonomy and a theory. Research Policy, $13,343-373$.

Peirce, C. S., (1867/1965). Collected papers of Charles Sanders Peirce. C. Hartshorne \& P. Weiss (Eds.), 1-6, Cambridge (MA) US: Harvard University Press.

Richiardi, M., Leombruni, R., Saam, N., \& Sonnessa, M. (2006). A common protocol for agent-based social simulation. Journal of Artificial Societies and Social Simulation, 9(1).

Schwerin, J. (2001). Wachstumsdynamik in Transformationsökonomien. Strukturähnlichkeiten seit der Industriellen Revolution und ihre Bedeutung für Theorie und Politik, Böhlau Verlag, Köln, Weimar, Wien.

Tsionas, E. G. (2000). Bayesian model comparison by Markov chain simulation: Illustration using stock market data. Research in Economics, 54, 403-416.

Thagard, P. (1988). Computational philosophy of science. Cambridge (MA) US: MIT Press.

Werker, C. (2003). Market performance and competition: A product life cycle model. Technovation, 23, 281-290.

Werker, C., \& Brenner, T. (2004). Empirical calibration of simulation models. Papers on Economics \& Evolution \#0410, Max Planck Institute of Economics, Jena.

Windrum, P., \& Birchenhall, C. (1998). Is life cycle theory a special case? Dominant designs and the emergence of market niches through co-evolutionary learning. Structural Change and Economic Dynamics, 9, 109-134.

Winter, S.G., Kaniovski, Y. M., \& Dosi, G. (2003). A baseline model of industry evolution. Journal of Evolutionary Economics, 13, 355-383.

Zellner, A. (1971). An introduction to Bayesian inference in econometrics. New York: Wiley. 